Measurement of mechanical loss in the Acktar Black coating of silicon wafers

This content has been downloaded from IOPscience. Please scroll down to see the full text.

2016 Class. Quantum Grav. 33185002

(http://iopscience.iop.org/0264-9381/33/18/185002)

View the table of contents for this issue, or go to the journal homepage for more

Download details:

IP Address: 131.215.248.4

This content was downloaded on 23/08/2016 at 18:41

Please note that terms and conditions apply.

You may also be interested in:

Mechanical losses of oscillators fabricated in silicon wafers

L G Prokhorov and V P Mitrofanov

Suppression of parametric instabilities in future gravitational wave detectors

S Gras, D G Blair and C Zhao

Coherent cancellation of photothermal noise in $\mathrm{GaAs} / \mathrm{Al}$ 0.92Ga0.08As Bragg mirrors

Tara Chalermsongsak, Evan D Hall, Garrett D Cole et al.

Broadband measurement of coating thermal noise in rigid Fabry-Pérot cavities

Tara Chalermsongsak, Frank Seifert, Evan D Hall et al.

Enhanced characteristics of fused silica fibers using laser polishing

A Heptonstall, M A Barton, A S Bell et al.

Experimental measurements of mechanical dissipation in dielectric coatings

D R M Crooks, G Cagnoli, M M Fejer et al.

Experimental measurements of coating mechanical loss factors

D R M Crooks, G Cagnoli, M M Fejer et al. 


\title{
Measurement of mechanical loss in the Acktar Black coating of silicon wafers
}

\author{
M R Abernathy ${ }^{1}$, N Smith ${ }^{1}$, W Z Korth ${ }^{1}, \mathbf{R}$ X Adhikari $^{1}$, \\ L G Prokhorov ${ }^{2}$, D V Koptsov ${ }^{2}$ and V P Mitrofanov ${ }^{2}$ \\ ${ }^{1}$ LIGO Laboratory, California Institute of Technology, Pasadena, CA 91125, USA \\ ${ }^{2}$ Faculty of Physics, Lomonosov Moscow State University, Moscow 119991, Russia \\ E-mail: prokhorovlg@phys.msu.ru and mitr@hbar.phys.msu.ru
}

Received 6 May 2016, revised 13 July 2016

Accepted for publication 29 July 2016

Published 22 August 2016

\begin{abstract}
Some proposed interferometric gravitational wave detectors of the next generation are designed to use silicon test masses cooled to cryogenic temperatures. The test masses will need to be partially coated with high emissivity coating to provide sufficient cooling when they absorb the laser light. The mechanical loss of the Acktar Black coating is determined based on the measurements of the $Q$-factors of the bending vibration modes of coated and uncoated commercial silicon wafers. The Young's modulus of the coating material is determined using nanoindentation. We use this information to calculate thermal noise of the silicon test masses associated with a high emissivity coating on its lateral side (barrel). It is found that such a coating results in a less than $9 \%$ increase of the total strain noise of LIGO Voyager design for a future cryogenic gravitational wave detector.
\end{abstract}

Keywords: interferometric gravitational-wave detectors, high-emissivity coating, mechanical loss, silicon test mass

(Some figures may appear in colour only in the online journal)

\section{Introduction}

On 14 September 2015 the two detectors of the laser interferometer gravitational-wave observatory (LIGO) detected a signal from the binary black hole merger GW150914 [1]. The detectors continue their observations and the next generation of detectors is under discussion $[2,3]$. Single crystalline silicon test masses suspended by silicon ribbons and kept at low temperature are considered prospective candidates for the third generation of GW detectors due to silicon's excellent mechanical, thermal and optical properties at a wavelength of about 
$1.5 \mu \mathrm{m}[2,4,5]$. The detector's operation at the temperature of about $123 \mathrm{~K}$ allows for significant reduction of the thermoelastic noise of the test masses due to the zero crossing in thermal expansion at this temperature [6-8]. In the proposed LIGO Voyager design the initial test mass cooldown will be done by means of the exchange gas or through copper braids [5]. During the detector operation the test mass needs to be kept at a temperature of about $123 \mathrm{~K}$. Therefore, it will be necessary to extract up to $8 \mathrm{~W}$ of heat absorbed by the test mass from the laser beam under high vacuum conditions [5]. This can be done by means of radiative cooling. However, the thermal emissivity of the silicon test mass and the optical coatings deposited upon its face is too small to provide the necessary rate of radiative cooling [9]. The emissivity of a barrel of a cylindrical test mass can be increased by using a high-emissivity coating such as DLC-Diamond-Like Carbon coating [10] or Acktar Black coating [11]. However, any coating of the test mass introduces additional mechanical loss and additional thermal noise associated with this loss. Knowing the coating loss angle is crucial to calculating the coated test mass thermal noise in order to optimize the coating parameters. The mechanical loss of a coating is usually determined by measuring a difference between the loss $Q^{-1}$ of the vibration modes of coated and uncoated substrates. Bending modes of thin cantilevers and disks are widely used for these measurements because the loss in a coating has a greater effect on the total loss as the substrate thickness is reduced $[12,13]$.

In this paper, we report the results of investigation of the mechanical loss in the Acktar Black coating on silicon wafers in the temperature range of 100-300 K. The loss is determined from the measured $Q$-factors of bending vibration modes of coated and uncoated commercial silicon wafers. The Young's modulus of the coating material is determined using nanoindentation. Based on these measurements we calculate the silicon test mass thermal noise associated with the Acktar Black coating of its barrel.

\section{Experimental measurements}

The Acktar Ultra Black ${ }^{\mathrm{TM}}$ coating was chosen from other types of Acktar coating because it provides high emissivity of about 0.85 at temperatures near $120 \mathrm{~K}$ [14]. Acktar Black coating is manufactured using a vacuum deposition technology. It is completely inorganic with a very high specific surface area, has low outgasing and low reflectivity over a wide wavelength range, broad working temperature range from 4 to $600 \mathrm{~K}$. The manufacturer declares the coating thickness tolerance \pm 0.6 micron [15]. The Acktar coating was deposited on one face of a commercial (Virginia Semiconductor Inc.) double side polished silicon (111) wafer (the electrical resistivity $>5000 \mathrm{ohm} \mathrm{cm}$ ) with diameter of $76.2 \mathrm{~mm}$ and thickness of $0.38 \mathrm{~mm}$. Young's modulus, Poisson ratio, and shear modulus are transversely and vertically isotropic for silicon (111) wafers [16]. High resistivity wafers are chosen because they contain fewer impurities that can cause an additional loss. The only available in-stock wafers have two flats indicating the crystallographic planes. To save time and to investigate whether the commercial wafers with flats can be used for the research we have ordered these in-stock wafers.

Temperature dependences of resonant frequencies and the mechanical loss $Q^{-1}$ of a number of bending vibration modes of the wafer are measured. The selected modes have different number of nodal diameters and zero nodal circles. The approximate resonant frequencies of these modes are: $1.3 \mathrm{kHz}, 3.6 \mathrm{kHz}, 5.1 \mathrm{kHz}, 8.8 \mathrm{kHz}(3,5,6$ and 8 nodal diameters respectively). Such modes have a nodal area at the center of the wafer and their vibrations are concentrated in the circumferential region of the wafer [17]. This reduces the clamping loss and allows us to obtain high $Q$-factors of these modes despite the presence of flats that slightly distort the spatial symmetry of distribution of the wafer vibration. The 


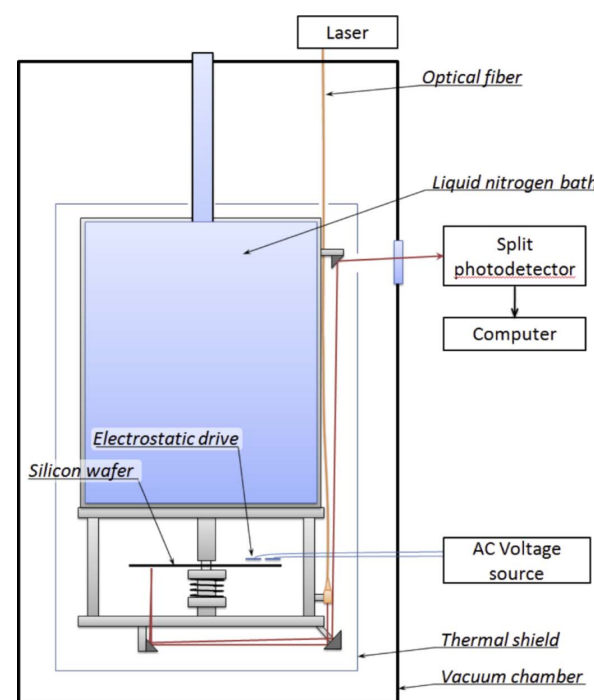

(a)
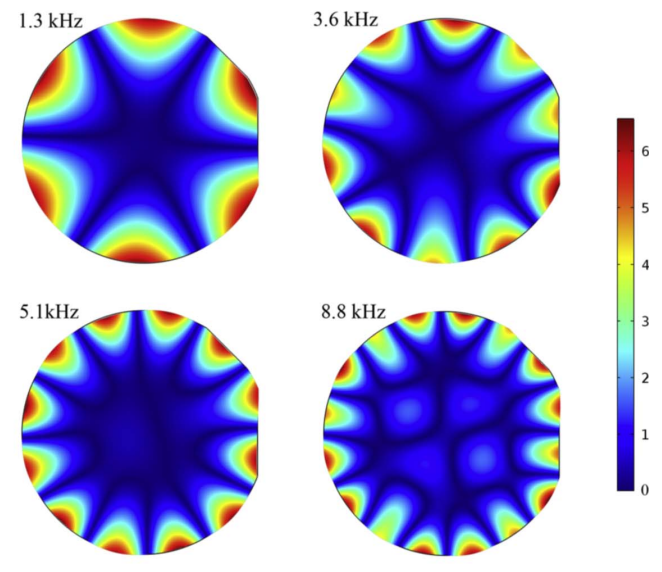

(b)

Figure 1. Schematic of the experimental setup (a). The distribution of the total displacement for the bending vibration modes with different numbers of nodal diameters calculated for the silicon wafer with two flats (b).

distributions of the total displacement calculated for the the four test modes of the wafer with two flats are shown in figure 1(b).

The schematic of the experimental setup is shown in figure 1(a). The silicon wafer is centrally clamped between two stems attached to an aluminum frame with a PTFE disk ( $8 \mathrm{~mm}$ diameter and $2 \mathrm{~mm}$ thickness) at each side of the wafer as a gasket. PTFE is used as a soft material in order to minimize the risk of breaking or scratching the wafer. One of the PTFE gaskets is mounted on the aluminum stem, another one is on the spring-loaded disk. The clamping force of about $10 \mathrm{~N}$ is set at the room temperature by adjusting the spring compression by means of the nut. Such a force provides a low level of clamping loss and is used for all investigated wafers. The spring stabilizes the clamping force when the temperature changes. This method of mounting at the central node is similar to another technique generally referred to as a gentle nodal suspension [18]. However, the gentle nodal suspension is not robust enough to hold the wafer during transfer into the vacuum chamber. As a fortunate side-effect, our clamping method allows the placement of a thermocouple sensor in direct contact with the wafer.

The frame is attached to a vessel mounted inside the vacuum chamber and filled with liquid nitrogen. Cooling results in the change of the clamping force mostly due to the temperature change of the spring rigidity. Our estimation shows that the relative change of the clamping force is less than $10 \%$ in the temperature range from room temperature to $100 \mathrm{~K}$. Such a design allows us to obtain reproducible results in the whole temperature range. In centrosymmetric uncoated silicon wafers we obtained the best value of $Q=2.5 \times 10^{7}$ at the temperature of about $123 \mathrm{~K}$. The presence of flats cut in the wafer results in the increase of the displacement amplitudes in the central part of the wafer, especially for the higher-order modes of vibration. 
The wafer vibration is excited at the resonant frequency of a mode using an electrostatic drive which consists of two plate electrodes placed $2 \mathrm{~mm}$ above the wafer. For the applied voltage with an amplitude of $U_{\text {exc }}=300 \mathrm{~V}$, the electrostatic drive generates an attractive force with an amplitude of about $10^{-6} \mathrm{~N}$. This allows us to excite the vibration amplitude up to $10^{-8} \mathrm{~m}$ for the modes with frequencies of up to $10 \mathrm{kHz}$. The wafer vibration amplitude is monitored by an optical sensor. Local bending of the wafer produced by its vibration results in a deflection of the laser beam reflected from the peripheral region of the wafer surface. The reflected beam passes through a system of mirrors to steer the light and is detected by a split photodiode placed outside the vacuum chamber. The vibration modes are identified by comparing their measured resonant frequencies with those computed by finite element modeling (FEM). The mode quality factor $Q$ is determined from the ring-down time $\tau$ of the vibration mode according to the relation $Q=\pi f \tau$, where $f$ is the mode resonant frequency. Measurements are carried out in vacuum under residual pressure of about $10^{-6}$ Torr. The level of the residual gas damping is calculated using the known formulae for the bending mode of a plate in the case of the free molecular regime and taking into account the squeeze film air damping effect [19]. It gives a negligible contribution to the measured mechanical loss.

The temperature of the wafer is measured by a thermocouple attached to a thin copper foil disk which is placed between the wafer and the PTFE gasket in the clamp. Usage of long (about $0.5 \mathrm{~m}$ ), thin $(0.1 \mathrm{~mm})$ thermocouple wires reduces any additional thermal flux to the wafer via thermal conduction of the wires. Measurements of the temperature dependence of $Q$-factors of the modes were made during the wafer cooling after the vessel is filled with liquid nitrogen or during the wafer's return to room temperature after the evaporation of the liquid nitrogen. In the wafer cooling regime, the cooling rate while close to the room temperature is found to be about $3 \mathrm{~K} \mathrm{~min}^{-1}$ for the uncoated silicon wafer and $6 \mathrm{~K} \mathrm{~min}^{-1}$ for the coated wafer. At temperatures of about $120 \mathrm{~K}$ the cooling rate is found to be about $0.2 \mathrm{~K} \mathrm{~min}^{-1}$ and $0.4 \mathrm{~K} \mathrm{~min}^{-1}$ respectively. In the wafer heating regime the heating rate decreases with increasing temperature. Relatively small differences in the cooling rate of coated and uncoated wafers is a consequence of a complex structure of the heat transfer between the wafer and surroundings. In particular, we did not use high emissivity coating for surfaces surrounding the wafer which are at temperatures close to the temperature of liquid nitrogen because the small cooling rate is required in order to get the state of the wafer close to the thermal equilibrium. Variation of the wafer's temperature during the measurement of the ring-down time did not exceed $0.5 \mathrm{~K}$. The temperatures near $120 \mathrm{~K}$ are of most interest, so our goal was to have a minimal rate of the temperature change and a minimal uncertainty of the measured temperature in this range. The uncertainty of the measured temperature was estimated to be $\pm 5 \mathrm{~K}$.

Our measurements were made using three wafers coated by the manufacturer. Initially we planned to measure mechanical loss in two coated silicon wafers, and to leave a third one as a reserve. Our study was mainly focused on the measurement of the Acktar Black coating loss at temperatures near $123 \mathrm{~K}$. It is crucial for the LIGO Voyager Project. The measured loss values were in the range $(2-3) \times 10^{-5}$ for all four investigated modes in the coated wafers $\# 1$ and \#2. The two coated wafers had then been destroyed for the destructive analysis of the coating thickness and Young's modulus. The average thickness of the coating layer of the wafer \#1 determined using scanning electron microscopy (SEM) is $18 \mu \mathrm{m}$. The thickness variation we see is consistent with the tolerance declared by the manufacturer. Later we decided to measure the loss thoroughly in the whole range of temperatures. Therefore only the wafer \#3 is studied more completely. 


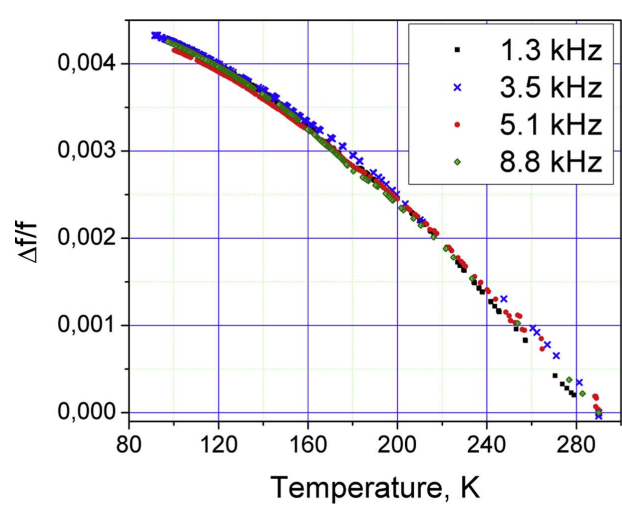

(a)

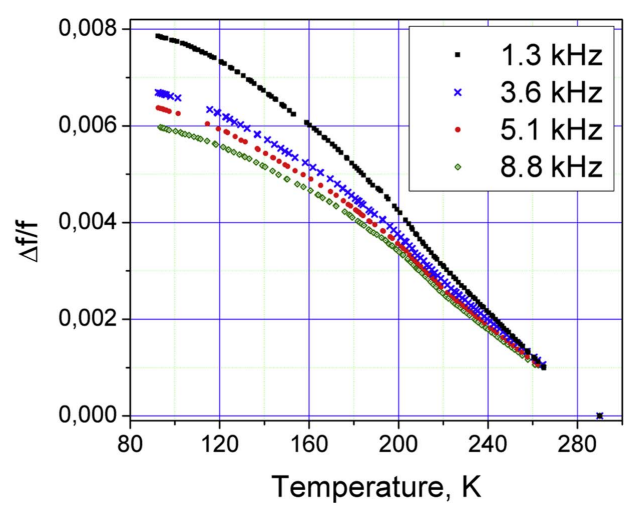

(b)

Figure 2. The temperature dependence of the relative change of the mode frequency for the uncoated silicon wafer (a) and the coated wafer \#3 (b).
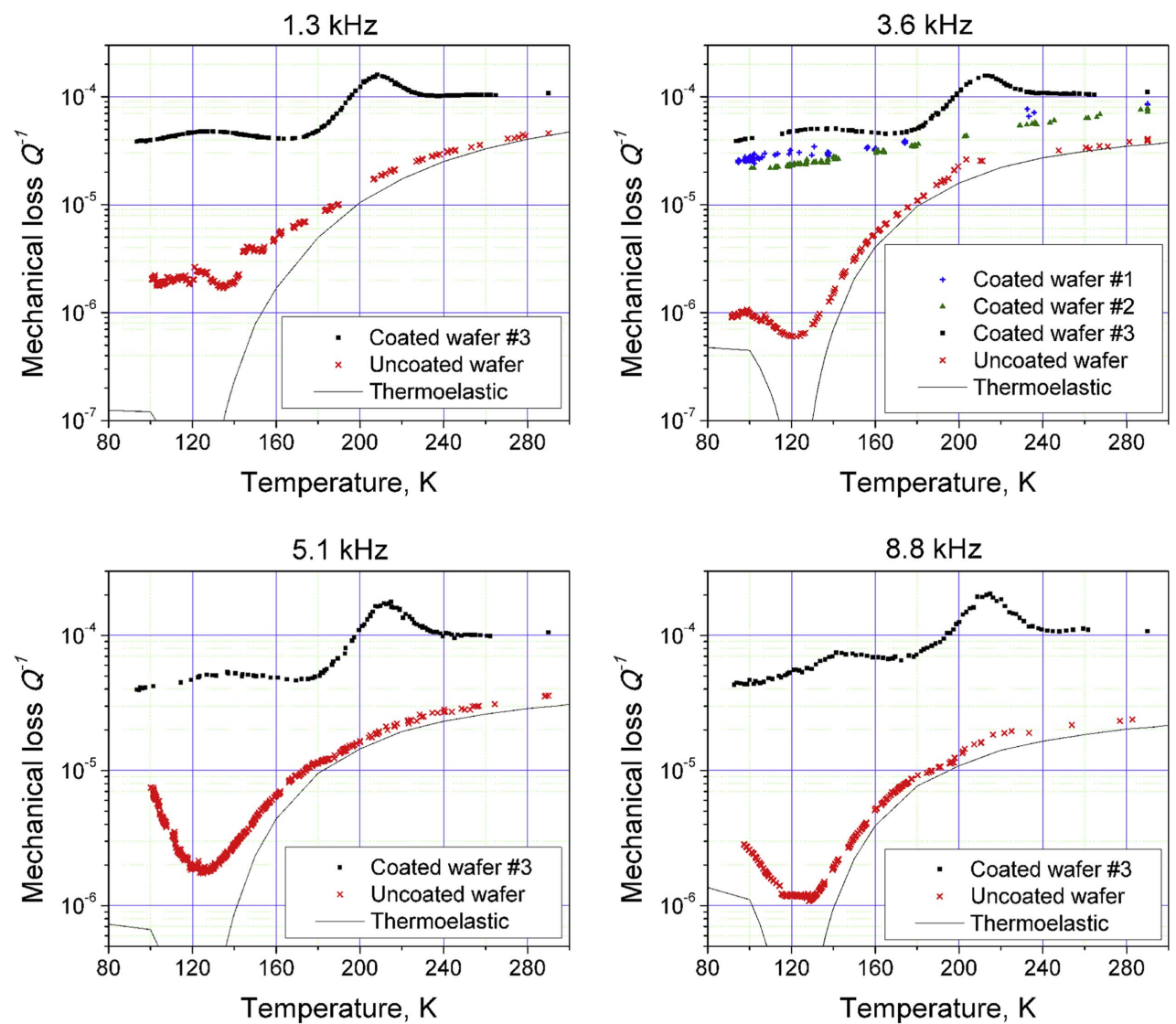

Figure 3. The temperature dependence of mechanical loss of different modes of coated and uncoated wafers. 


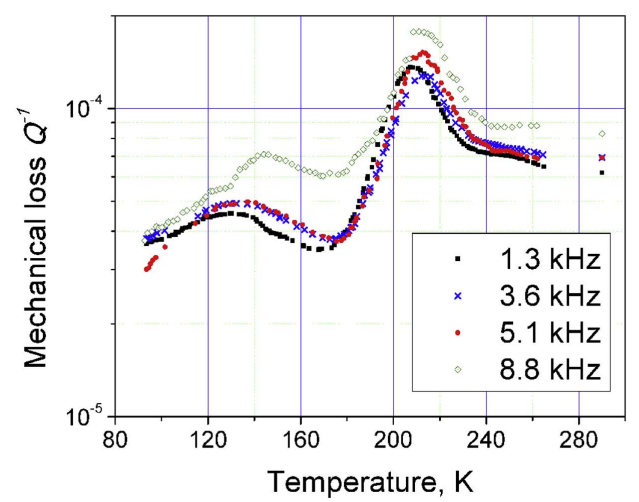

(a)

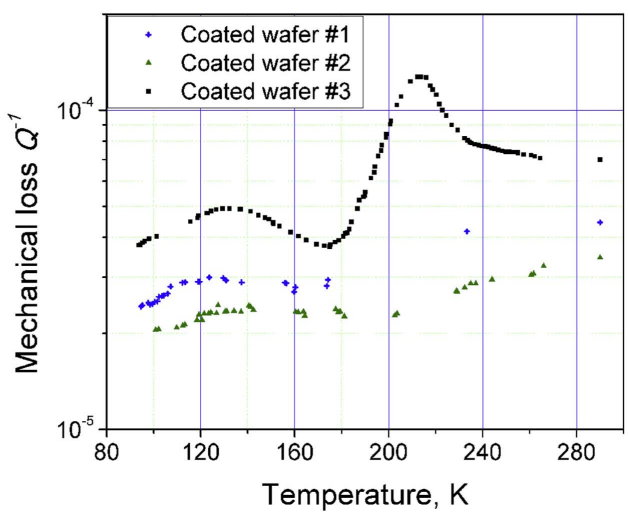

(b)

Figure 4. The differences in the loss measured in the coated wafer and the uncoated wafer (a) for different modes of the coated wafer \#3, (b) for the $3.6 \mathrm{kHz}$ mode of the coated wafers $\# 1, \# 2, \# 3$.

The temperature dependence of the relative change of the mode frequency $\frac{f(T)-f\left(T_{o}\right)}{f\left(T_{o}\right)}$, where $T_{o}=293 \mathrm{~K}$, measured for four vibration modes of the uncoated silicon wafer is shown in figure 2(a). It is the result of the temperature-dependent variation in geometrical parameters (radius and thickness) of the wafer as well as the density, Young's modulus and Poisson ratio of silicon. The Young's modulus variation plays a major role [20]. For the coated wafer \#3 the temperature dependence of the relative change of the mode frequency measured for the same vibration modes is shown in figure 2(b). The full temperature dependent change of the mode frequency in the temperature range 100-293 K is larger for the coated samples than for the uncoated ones. This difference increases with the decrease of the vibration mode frequency. Such behavior of the vibration mode frequencies can be explained by stresses introduced in the coating and the substrate due to differential thermal expansion between the film and the substrate. It is known that tensile axial loads change the resonant frequencies of the bending modes of beams [21]. Structure of vibration of the bending modes in a wafer is similar to that in a thin beam, so one can expect the additional temperature change of the mode frequency due to the additional temperature induced stress. For the mode with a higher frequency, vibration is localized in the area closer to the peripheral region of the wafer where the stresses are likely to be smaller.

The temperature dependences of the loss $Q^{-1}$ measured in the uncoated wafer for four vibration modes are shown in figure 3 . In the uncoated wafer the loss reaches the minimal value at a temperature of about $123 \mathrm{~K}$ for each mode. The loss curves have a characteristic shape due to the suppression of the thermoelastic loss arising from the decrease in the thermal expansion coefficient of silicon. The level of the thermoelastic loss is shown with thin solid line. It is calculated using Comsol Multiphysics FEM software package [22]. The relative value of the difference between the measured and calculated loss is significant at low temperatures where the measured loss approaches the level of $10^{-6}$. We attribute this to the effect of the clamping loss. The clamping loss depends on the properties of the contact area between the surfaces of the wafer and the mount as well as on the clamping force. The clamping design providing the constant clamping force was used in order to obtain the same clamping conditions and clamping loss for the same modes of all wafers. This and the fact that the 


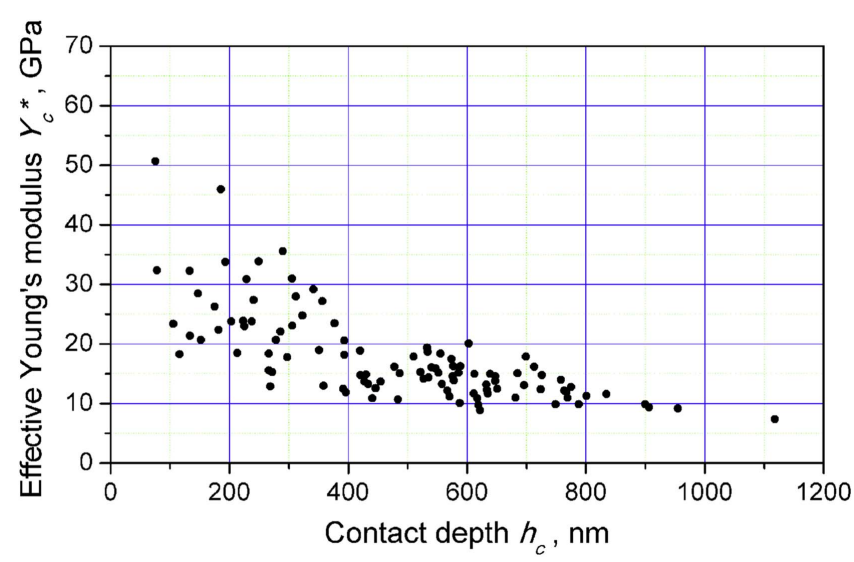

Figure 5. Effective Young's modulus of Acktar Black coating versus contact depth.

clamping loss is at least an order less than the loss in the coated wafer allowed us to neglect the difference in the clamping loss of coated and uncoated wafers.

The temperature dependences of the loss $Q^{-1}$ measured for the modes of the coated wafer \#3 are also shown in figure 3. The differences in the loss measured in the coated wafer \#3 and the uncoated wafer for different modes are summarized in figure 4(a). One can see two peaks in the temperature dependence of loss for each mode. The low temperature peak is more flat than the high temperature one. The temperature dependences of loss of the three modes are almost identical with exception of the peak position, which shifts with the mode frequency. Formally this indicates the presence of thermally activated relaxation processes resulting in the loss. But in this case one should be careful when using the theory of relaxation processes and calculating the activation energy and the rate constant which are related by the Arrhenius equation [23], as it may not be applicable to such a complex structure as the Acktar Black coating. The $8.8 \mathrm{kHz}$ mode has a higher level of loss. A plausible explanation is that the frequency of the $8.8 \mathrm{kHz}$ mode is close to one of the resonance frequencies of the clamping structure. This enhances the coupling between them and therefore the leakage of the energy from the wafer increases. The coupling and the loss can change with temperature, because the frequencies change with temperature.

Only the $3.6 \mathrm{kHz}$ mode has been measured for the wafers \#1 and \#2 in whole range of temperatures. The loss for the other modes is measured only at the room temperature and the temperatures near $123 \mathrm{~K}$. However, these data are interesting, because the loss levels of this mode measured in three coated wafers at temperatures near $123 \mathrm{~K}$ differ approximately by a factor of two (see figure 4(b)). It is important to note that this difference is not caused by the errors of the measurements of the loss which do not exceed $10 \%$.

We think that the difference in the loss measured for the three coated wafers, including the dissipation peak at $\approx 210 \mathrm{~K}$, and the higher loss of the wafer \#3 may be associated with variations of the coating thickness, damage of the wafer in the process of the coating fabrication and other factors specific to each wafer. In particular, there are marks on the wafers left by the mounting procedure for the coating deposition. The marks are weak but the mounting of the wafer can create microcracks which are a source of dissipation.

In order to calculate mechanical loss in the coating layer, the elastic parameters of the substrate and the coating material have to be known. Effective Young's modulus, $Y_{c}^{*}$, of the Acktar Black coating, composed of the coating and substrate Young's moduli [24], was 
measured using the Oliver and Pharr method of nanoindentation [25]. It was assumed that the Poisson ratio of the coating material is equal to that of the silicon, $\nu_{c}=\nu_{s}=0.27$ [26], which can lead to a small systematic uncertainty [24]. Measurements were made using a Hysitron Ubi indenter equipped with a diamond indentation tip [27]. It is a diamond Berkovich tip with a radius of curvature of $\approx 100 \mathrm{~nm}$ [28]. The tip area function was measured to be $A(h)=39.11 \times h^{2}+2.436 \times 10^{4} \times h^{1 / 4}$. Indentations were made at multiple locations on one coated wafer with maximum loads of 500-10000 N, yielding indentation depths of $0.1-1 \mu \mathrm{m}$. The results of measurements are shown in figure 5. Each point is one indent on the Acktar Black coating. One can find the detailed description of the method of the coating Young's modulus extraction from the measured values in [24]. Taking into account that the thickness of the coating is much smaller than the thickness of the substrate and greater than the contact depth of the indent we obtain $Y_{c} \approx Y_{c}^{*}$ [24]. The resulting Young's modulus values show heterogeneity throughout the surface thickness of the coatings with surface values as high as $50 \mathrm{GPa}$ and asymptotically approaching $10 \mathrm{GPa}$ at depths of $1 \mu \mathrm{m}$. It is difficult to understand and explain such a behavior of the modulus without knowing more about the manufacturing process of the material. As the regions with higher Young's moduli are small compared with the coating thickness, we take the value of $10 \pm 2 \mathrm{GPa}$ as an estimate for the Young's modulus of the Acktar Black coating. It is obtained as a result of averaging the data in the range from 800 to $1100 \mathrm{~nm}$. As will be shown in section 3 the effect of the uncertainty of the coating Young's modulus on the calculated power spectral density associated with the coating is weak. Additionally, the hardness of the coating was found to be $<1 \mathrm{GPa}$, which, while not necessary to thermal noise calculations, shows that the coating is soft and easily damaged.

\section{Calculation of the coating loss}

Assuming that the only non-negligible sources of mechanical loss are from the coating and the substrate, the value of the loss in the coating material, described by a loss angle $\phi_{\text {coating }}$, is calculated from the following equation [29]:

$$
\phi_{\text {coating }}=\frac{Q_{\text {coated }}^{-1}-Q_{\text {uncoated }}^{-1}}{t_{c} \mathrm{~d} U / U},
$$

where $t_{c}$ is the thickness of the coating layer and $\mathrm{d} U / U$ is the ratio of energy stored in the coating per unit coating thickness to the total energy for the given mode shape. The values of $\mathrm{d} U / U$ for different modes were calculated numerically using a finite element model developed in the Comsol Multiphisics. Taking into account that the calculated change of the wafer frequencies associated with the flats is about $1 \%$ for the lower-order modes and the variation of in-plane elastic parameters for typical commercial (111) Si wafers is about 6\% [30], we chose to use a simplified model for calculation of the coating loss in the silicon wafers, which will likely have an error less than that which is inherent to the measurement. An axisymmetric wafer fabricated from an isotropic material with mechanical properties equal to that of silicon along (111) plane was considered [26]. Due to rotational symmetry of the selected modes, the calculations were performed using a sector of the wafer and periodic boundary conditions. A triangular mesh was constructed on the surface between the wafer and the coating and then translated to the wafer and the coating using the 'mesh sweeping' process. This allowed us to overcome the difficulties associated with the large ratio of the sample diameter to the thickness of the coating. In order to assess the accuracy of the FEM model we have compared the mode frequencies obtained with FEM simulation and the 


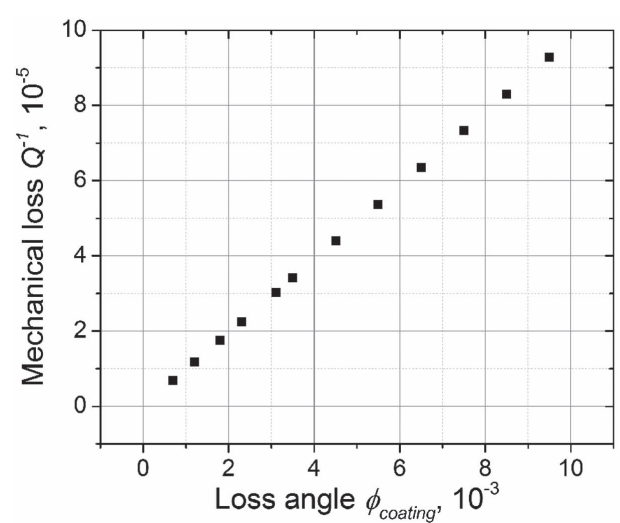

(a)

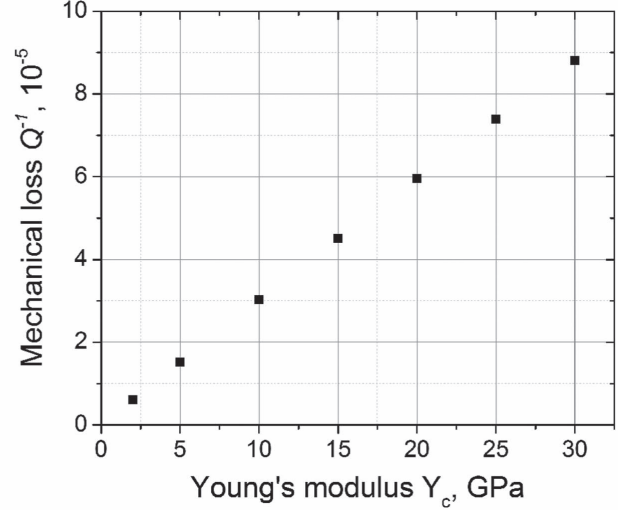

(b)

Figure 6. Mechanical loss of a coated wafer calculated using FEM simulation as a function of the coating loss angle (a), coating Young's modulus (b). Non-variable parameters are the following: $t_{c}=18 \mu \mathrm{m}, Y_{c}=10 \mathrm{GPa}, Y_{s}=156 \mathrm{GPa}$, $\nu_{s}=\nu_{c}=0.27, \phi_{c}=3.1 \times 10^{-3}$.

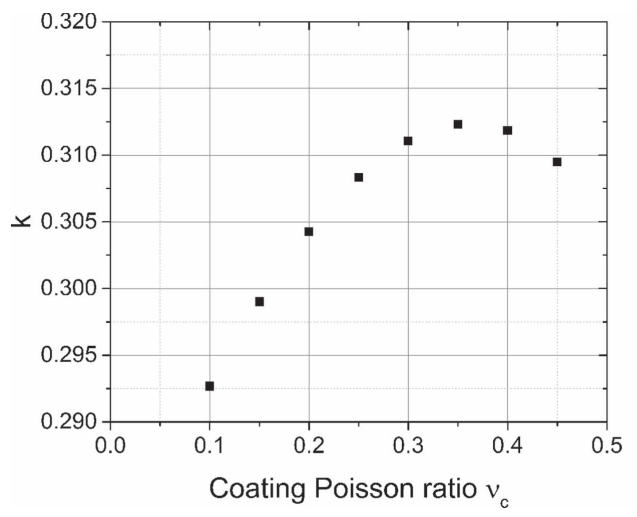

(a)

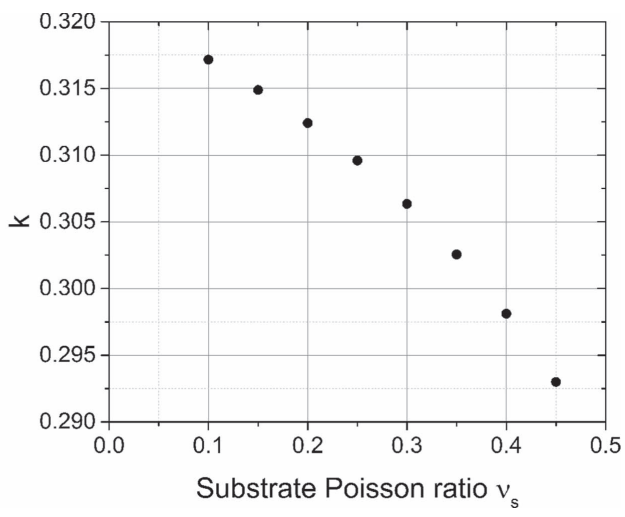

(b)

Figure 7. The coefficient $k$ calculated using FEM simulation as a function of the coating Poisson ratio $\nu_{c}(\mathrm{a})$, the substrate Poisson ratio $\nu_{s}$ (b). Non-variable parameters are the following: $\quad t_{c}=18 \mu \mathrm{m}, \quad Y_{c}=10 \quad \mathrm{GPa}, \quad Y_{s}=156 \quad \mathrm{GPa}$, $\nu_{s}=\nu_{c}=0.27, \phi_{c}=3.1 \times 10^{-3}$.

measured frequencies for the uncoated and coated wafers. The relative differences of these frequencies do not exceed 3\% for the values of the parameters indicated in the figure 6 caption. This error can be associated with the uncertainties of these parameters. The relative differences of the measured and computed ratios of the mode frequencies to the frequency of the $1.3 \mathrm{kHz}$ mode do not exceed $1 \%$. This value may be considered as an estimate of the model accuracy. Results of FEM simulation performed for the $3.6 \mathrm{kHz}$ mode assuming $Q_{\text {uncoated }}^{-1}=0$ are presented in figure 6 .

One can see that the loss of the coated wafer $Q_{\text {coated }}^{-1}$ associated with the loss in the coating is approximately a linear function of the coating loss angle $\phi_{\text {coating }}$ and Young's modulus of 
the coating material $Y_{c}$ in the considered range of parameters. The dependence on the coating layer thickness $t_{c}$ is also close to linear in the range of thickness values 2-24 $\mu \mathrm{m}$. Based on this fact, the mechanical loss angle of the coating can be written as a function of the difference between the measured loss of the bending modes of the coated and uncoated wafers:

$$
\phi_{\text {coating }}=k \frac{Y_{s} t_{s}}{Y_{c} t_{c}}\left(Q_{\text {coated }}^{-1}-Q_{\text {uncoated }}^{-1}\right)
$$

where $Y_{s}$ and $t_{s}$ are Young's modulus and thickness of the substrate material. The coefficient $k$ is obtained from the FEM simulation. It varies within $1 \%$ range for the modes with the number of nodal diameters from 3 to 12 . The equation (2) coincides with the one describing the similar system in [31]. The main source of error in the computation of the coefficient $k$ is the unknown Poisson ratio of the Acktar Black coating material. Figure 7 shows the computed dependence of the coefficient $k$ on the Poisson ratio of the coating material $\nu_{c}$ and of the substrate Poisson ratio $\nu_{s}$. One can see that within the range of Poisson ratio typical values $0.1<\nu_{c}<0.5$ the relative value of coefficient $\mathrm{k}$ changes within $7 \%$. Therefore we can determine $k=0.305 \pm 0.015$.

There is more than a factor of two difference between $Q_{\text {coated }}^{-1}-Q_{\text {uncoated }}^{-1}$ measured across three wafers in the temperature range near $123 \mathrm{~K}$ (see figure 4(b)). Provided that the thicknesses and Young's moduli are the same for these three wafers, the calculated values of $\phi_{\text {coating }}$ of these wafers differ by the same factor. As discussed above this may be the result of unknown factors associated with the coating fabrication. For the coated wafer \#1 all values of $\phi_{\text {coating }}$, calculated for modes $1.3,3.6$ and $8.8 \mathrm{kHz}$ at the temperature near $123 \mathrm{~K}$ are in the limits of $(3.1 \pm 0.3) \times 10^{-3}$. This value was used for the calculation of thermal noise associated with the Acktar Black coating.

Notice that the coating loss calculated from (2) can include the internal friction loss and the thermoelastic loss. In order to calculate the latter, one needs to know the thermal properties of the coating material, which are not available. Since the thermoelastic loss strongly depends on temperature and frequency, whereas the measured coating loss weakly depends on these parameters, we can conclude that the contribution of the thermoelastic loss is small.

Thermal Brownian noise associated with the Acktar Black coating of the silicon test mass barrel is calculated based on Levin's approach which involves the direct application of the fluctuation-dissipation theorem to the interferometer readout of the position of the test mass face $[32,33]$. An axisymmetric isotropic model of the test mass with Young's modulus $Y_{s}=156 \mathrm{GPa}$ and Poisson ratio $\nu_{s}=0.27$ is considered (i.e. no bonding elements have been modeled). The silicon test mass is represented by a cylinder with diameter of $45 \mathrm{~cm}$ and thickness of $55 \mathrm{~cm}$. It has an amorphous silicon/silica high reflective optical coating on the front face. Mechanical parameters of the coating materials are taken from [5]. Difference between coating layers materials is neglected and average values of Young's modulus, Poisson ratio and loss factor are taken. The coating thickness considered in the model is the sum of all layers and is equal to $3.27 \mu \mathrm{m}$ for the end test mass (ETM) and $1.96 \mu \mathrm{m}$ for the intermediate test mass (ITM). The barrel is coated with $18 \mu \mathrm{m}$ of the Acktar Black coating. A pressure with the Gaussian distribution with $8.4 \mathrm{~cm}$ (ETM) and $5.9 \mathrm{~cm}$ (ITM) radius (i.e. the radius at which the laser beam intensity is $1 / e^{2}$ of the maximum intensity) is applied to the face with the optical coating. In order to compensate this pressure, an evenly distributed countervailing volume force is applied to the test mass. The center of the rear face of the test mass is fixed in order to remove the translational degree of freedom. The values of the strain energy stored in the substrate, the high reflective coating and the barrel coating are computed numerically using Comsol Multiphysics. Then the three corresponding values of the power spectral densities of thermal displacement noise are calculated according to the fluctuation- 


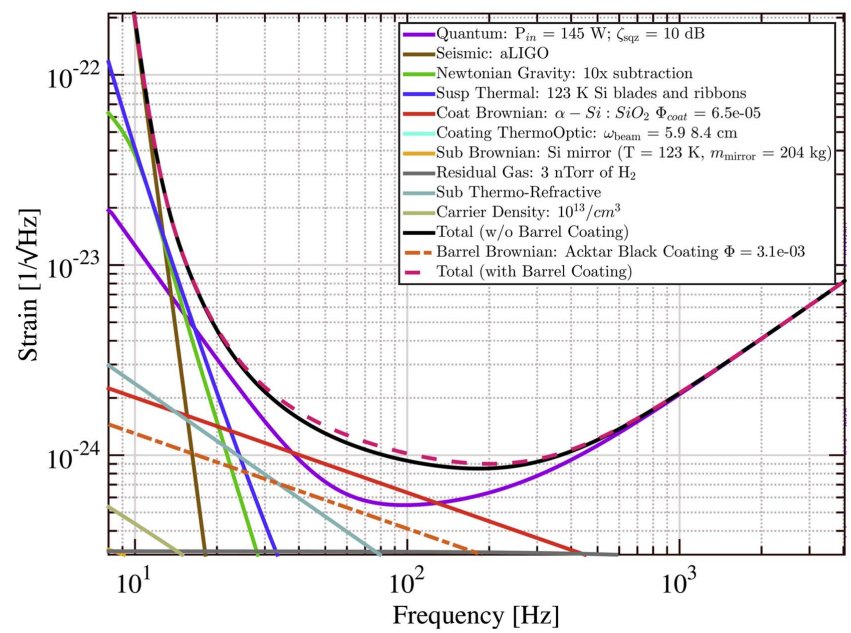

Figure 8. Noise budget for LIGO Voyager future gravitational wave detector [5] with calculated Brownian noise of the barrel Acktar Black coating.

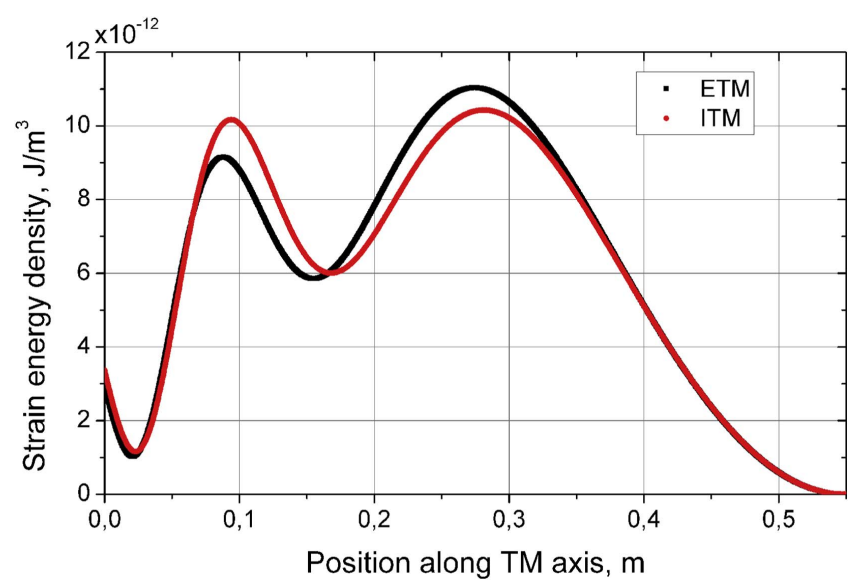

Figure 9. Strain energy density stored in the barrel coating as a function of distance from the test mass front face.

dissipation theorem [32]. The value of thermal displacement noise for the substrate was compared to the one computed by the GWINC package [26] and the relative difference was found to be less than $2 \%$.

It has been found that the noise power spectral density is proportional to the barrel coating thickness and Young's modulus when the thickness is in the range of $10-40 \mu \mathrm{m}$ and Young's modulus is in the range of 5-50 GPa. Therefore despite considerable uncertainty in the Young's modulus of the Acktar Black coating, we are able to estimate the noise level with the same error as the loss in the coated wafer (10\%). The noise budget of the future cryogenic gravitational wave detector LIGO Voyager is presented in figure 8 [5]. The calculated level of Brownian thermal noise of the Acktar Black barrel coating is shown with the orange dash-dot line. The result of appending the barrel coating noise to the total strain noise is shown with the dashed line. With the barrel coating the total strain noise increases by no more than $9 \%$. The 
influence of this additional noise source is maximal around $100 \mathrm{~Hz}$. The distribution of strain energy density stored in the barrel coating as a function of distance from the test mass front face is shown in figure 9. The shape of this distribution allows optimization of the coating by covering only the areas where the strain energy density is minimal.

\section{Conclusion}

The high emissivity Acktar Black coating deposited on the barrel surface of the silicon test mass of the future cryogenic interferometric gravitational wave detectors can provide extraction of heat from absorbed laser power. We have determined the mechanical loss of the Acktar Black coating with a thickness of $18 \mu \mathrm{m}$ based on measurements of the mechanical loss of bending vibration modes of coated and uncoated commercial silicon wafers. The Young's modulus of the coating material has been determined using nanoindentation. Its values show heterogeneity throughout the thickness of the coatings with surface values as high as $50 \mathrm{GPa}$ and asymptotically approaching $10 \pm 2 \mathrm{GPa}$ at depths of $1 \mu \mathrm{m}$.

The losses measured in three coated wafers at temperatures near $123 \mathrm{~K}$ differ approximately by a factor of two. Such a difference is likely caused by features of the coating process. The coating loss of $3.1 \times 10^{-3}$ obtained from the loss measurements of three modes of the coated wafer \#1 which had the average level of loss were used for the calculation of the silicon test mass thermal noise caused by the Acktar Black coating of the barrels in the LIGO Voyager detector design. According to the above calculations, a uniform Acktar Black coating applied to the test mass barrel would result in an approximately $9 \%$ increase of the total strain noise. The large scatter in the measured values of the coating's mechanical loss indicates that optimization of deposition parameters may reduce this value. Analysis and improvement of the coating fabrication process are required.

\section{Acknowledgments}

The authors would like to thank Stan Whitcomb for his help, Ian Martin and Peter Murray for the valuable comments, Denis Presnov who made the SEM image of the coating. This work was supported by the Russian Foundation for Basic Research under grant 14-02-00399 and the United States National Science Foundation under grant PHY-1305863. LIGO was constructed by the California Institute of Technology and Massachusetts Institute of Technology with funding from the National Science Foundation and operates under cooperative agreement PHY-0757058.

\section{References}

[1] Abbott B P et al 2016 Observation of gravitational waves from a binary black hole merger Phys. Rev. Lett. 116061102

[2] Abernathy M et al (ET science team) 2011 Einstein gravitational wave telescope conceptual design study ET-0106C-10 (www.et-gw.eu/etdsdocument)

[3] McClelland D 2015 Instrument science white paper LIGO Technical Note T1500290 (https://dcc. ligo.org/LIGO-T1500290/public)

[4] Adhikari R X 2014 Gravitational radiation detection with laser interferometry Rev. Mod. Phys. 86 121-51

[5] Adhikari R et al 2016 LIGO voyager upgrade conceptual design LIGO Technical Note T1400226-v7 
[6] Swenson C A 1983 Recommended values for the thermal expansivity of silicon from 0 to $1000 \mathrm{~K}$. J. Phys. Chem. Ref. Data 12 179-82

[7] Rowan S et al 2003 Test mass materials for a new generation of gravitational wave detectors Proc. SPIE $4856292-7$

[8] Cumming A V et al 2014 Silicon mirror suspensions for gravitational wave detectors Class. Quantum Grav. 31025017

[9] Weiss R 2012 Silicon mirror cooled to 120 K LIGO Technical Note T1200093

[10] Sakakibara Y et al 2014 A study of cooling time reduction of interferometric cryogenic gravitational wave detectors using a high-emissivity coating AIP Conf. Proc. 1573 1176-83

[11] Acktar Ltd. Black coating services http://acktar.com/category/BlackOpticalCoating

[12] Murray P G et al 2015 Ion-beam sputtered amorphous silicon films for cryogenic precision measurement systems Phys. Rev. D 92062001

[13] Hirose E et al 2014 Mechanical loss of a multilayer tantala/silica coating on a sapphire disk at cryogenic temperatures: toward the KAGRA gravitational wave detector Phys. Rev. D 90 102004

[14] Kralik T and Katsir D 2009 Black surfaces for infrared, aerospace, and cryogenic applications Proc. SPIE 7298 1-9

[15] Acktar Ltd. Acktar black world's blackest coatings http://acktar.com/files/pdf/Acktar_ brochure.pdf

[16] Kim J, Dong-il (Dan) C and Muller R S 2001 Why is (111) Silicon a Better Mechanical Material for MEMS? (Berlin: Springer) pp 662-5

[17] Dmitriev A V, Gritsenko D S and Mitrofanov V P 2014 Non-axisymmetric flexural vibrations of free-edge circular silicon wafers Phys. Lett. A 378 673-6

[18] Cesarini E, Lorenzini M, Campagna E, Martelli F, Piergiovanni F, Vetrano F, Losurdo G and Cagnoli G 2009 A 'gentle' nodal suspension for measurements of the acoustic attenuation in materials Rev. Sci. Instrum. 80053904

[19] Bao M and Yang H 2007 Squeeze film air damping in MEMS Sensors Actuators A 136 3-27

[20] Gysin U et al 2004 Temperature dependence of the force sensitivity of silicon cantilevers Phys. Rev. B 69045403

[21] Bokaian A 1990 Natural frequencies of beams under tensile axial loads J. Sound Vib. 142 481-98

[22] COMSOL Multiphysics: the platform for physics-based modeling and simulation https://comsol. com/comsol-multiphysics

[23] Nowick A S and Berry B S 1972 Anelastic Relaxation in Crystalline Solids (New York: Academic)

[24] Abernathy M R et al 2014 Investigation of the Young's modulus and thermal expansion of amorphous titania-doped tantala films Appl. Opt. 53 3196-202

[25] Oliver W C and Pharr G M 2004 Measurement of hardness and elastic modulus by instrumented indentation: advances in understanding and refinements to methodology J. Mater. Res. 19 3-20

[26] Advanced LIGO Gravitational wave interferometer noise calculator https://awiki.ligo-wa.caltech. edu/aLIGO/GWINC.

[27] Hysitron ubi-1 nanoindenter https://hysitron.com/

[28] Fischer-Cripps A C 2010 The sharpness of a berkovich indenter J. Mater. Res. 25 927-34

[29] Harry G M et al 2007 Titania-doped tantala/silica coatings for gravitational-wave detection Class. Quantum Grav. 24405

[30] França D R and Blouin A 2004 All-optical measurement of in-plane and out-of-plane Young's modulus and Poisson's ratio in silicon wafers by means of vibration modes Meas. Sci. Technol. 15859

[31] Yamamoto $\mathrm{K}$ et al 2006 Measurement of the mechanical loss of a cooled reflective coating for gravitational wave detection Phys. Rev. D 74022002

[32] Levin Yu 1998 Internal thermal noise in the LIGO test masses: a direct approach Phys. Rev. D 57 659-63

[33] Coyne D and Willems P 2008 Thermal noise increase due to a gold coated barrel LIGO Technical Note T080003 (https://dcc.ligo.org/T080003/public) 